\title{
REDUCTIONS OF RESIDUALS ARE FINITE
}

\author{
BY
}

R. HINDLEY

\begin{abstract}
An important theorem of the $\lambda \beta K$-calculus which has not been fully appreciated up to now is D. E. Schroer's finiteness theorem (1963), which states that all reductions of residuals are finite.

The present paper gives a new proof of this theorem and extends it from $\lambda \beta$-reduction to $\lambda \beta \eta$-reduction and reductions with certain extra operators added, for example the pairing, iteration and recursion operators. Combinatory weak reduction, with or without extra operators, is also included.
\end{abstract}

1. Introduction. In their original proof of the Church-Rosser theorem in [2], Church and Rosser proved, besides the main theorem, two other basic lemmas. One of these is now widely known (well, known to $\lambda$-calculus enthusiasts anyway); it says that all complete reductions of the residuals of a fixed set of redexes have the same end. This has been discussed by Curry and Feys in [4] as property E, p. 113, and further investigated in [8], and there are several independent proofs of it in the literature.

But the other lemma has been almost completely ignored. It says that no reduction of the residuals of a set of redexes can be infinitely long. Though Church and Rosser only proved this for the restricted system called nowadays $\lambda I \beta$-reduction, D. E. Schroer later carried out the (definitely nontrivial) extension of this result to the full $\lambda \beta$-system, in his thesis [12, Theorem 6.20].' Unfortunately Schroer's thesis has not been published yet, and I think many people still regard the finiteness property (if they remember it at all) merely as a peculiarity of the restricted $\lambda I$-calculus.

In fact the property is much more than that. All the proofs of the Church-Rosser theorem for $\lambda$-calculi, slick or clumsy, turn out to be based on reductions of residuals, and the finiteness property is one of the two main underlying facts which make all such proofs work. (The other one is of course what Curry and Newman call 'Property D', the Church-Rosser property for one-step reductions; see Lemma 2 below. $)^{2}$

Received by the editors June 8, 1976 and, in revised form, January 7, 1977.

AMS (MOS) subject classifications (1970). Primary 02C20, 68A05.

Key words and phrases. Reduction, residuals, relative reduction, development, lambda-calculus, combinators, finiteness-of-developments theorem.

'Though [12] was not completed until 1965, a preliminary version was available in 1963.

${ }^{2}$ Unfortunately the known proofs of the finiteness property are no simpler than the best proofs of the Church-Rosser theorem, so the above remark will not simplify any Church-Rosser proofs. 
Also, the finiteness property has turned out to be needed in Curry and Feys' proof of the standardization theorem for $\lambda \beta$-reduction [4, pp. 140-142], though this need does not seem to have been noticed in [4]. This point will be amplified in [9].

The present paper will give a proof of the finiteness of reductions of residuals for $\lambda K \beta$-reduction, based on the method of Church and Rosser's original $\lambda I$-proof. (Reductions of residuals will be called developments, following Schroer.) The proof will then be extended to $\beta \eta$-reduction, combinatory weak reduction, and reductions generated by the recursion operator and other similar operators.

Schroer's original proof was different from the one below. (In fact the proof below was made having forgotten the fact that Schroer's already existed, though I had seen Schroer's proof some years before.) Schroer's was carried out in an abstract setting which includes combinatory and $\lambda \beta$ reductions, and probably includes the recursion and similar operators to be described below, but not $\beta \eta$-reduction (though I have not checked this in detail).

Besides Schroer's proof and the one below, there is a nice proof by J. M. E. Hyland, in his manuscript [11], 1973. His is a proof for $\lambda \beta$-reduction, and it proceeds by assigning indices to sets $\alpha$ of redexes in such a way that contracting any member $\xi$ of $\alpha$ reduces the index of $\alpha$, with respect to a certain well-ordering. (I.e. the index of the set $\alpha / \xi$ of residuals is less than that of $\alpha$.) Hyland then uses the finiteness property to deduce property $E$ and hence the Church-Rosser theorem. (In contrast, Schroer's proof and the one in the present paper both depend on having proved $\mathrm{E}$ beforehand.)

The only other proof of the finiteness property that I know of at present is a very neat one in a manuscript by Barendregt, Bergstra, Klop and Volken [1, pp. 14ff., 20ff.]. This proof also uses indices, but the indexing-map is simpler than Hyland's, the indices are just positive integers, and the proof covers $\beta \eta$-reduction as well as $\beta$. It also covers extra reduction-rules $M \rightarrow \Omega$ where $M$ is any unsolvable closed term and $\Omega$ is one fixed unsolvable term, for example

$$
(\lambda x \cdot x x)(\lambda x \cdot x x)
$$

Like Hyland's, this proof does not depend on knowing property E beforehand.

The present paper was begun during a visit in 1975 to the Pennsylvania State University, whom I wish to thank for their very kind hospitality and financial support. I am also grateful to H. B. Curry for very helpful discussions and advice, leading to the present proof. Finally $I$ thank the referee for suggesting some improvements in the paper's exposition. 
2. Notation and basic lemmas. The reader is assumed to know already about $\lambda$-calculi, combinators and the basic notation of [4] or [10]. This paper will use the same notation, except that identity of terms will be denoted by '=' not ' $\equiv$ ', and reducibility will be denoted by ' $>$ ' (one step) or ' $\gg$ ' (several steps, possibly none). Changes of bound variables will be ignored throughout.

The usual type-restrictions may be included in the systems if desired; none of the results below will be affected.

Recall that the basic axioms for $\lambda$-reduction are

$$
\begin{gathered}
(\lambda x \cdot M) N>[N / x] M ; \\
\lambda x \cdot M x>M \quad \text { (if } x \text { is not free in } M) .
\end{gathered}
$$

In this paper the word 'axiom' will mean a particular instance of $(\beta)$ or $(\eta)$, for particular $M, N, x$.

As usual, a redex ( $\beta$ - or $\eta$-) will be the left-hand side of an axiom, and its contractum will be its corresponding right-hand side. However, in future the word 'redex' will almost always mean a particular occurrence of a redex in a term; for example 'all redexes in $X^{\prime}$ ' will mean 'all the occurrences of redexes in $X^{\prime}$.

To distinguish different occurrences of a redex in a term $X$, one can associate with each occurrence an index called a position. This is done in detail in [8] at the start of $\$ 6$. The only properties of positions we shall need here are that if $U$ occurs in $X$, then contracting a redex that does not overlap $U$ will leave the position of $U$ unchanged, and contracting a redex inside $U$ will also not change the position of $U$ (though it will change $U$ itself, of course). These properties are stated more fully in $[8, \S 6$, Let, na 0].

A contraction in a term $X$ is the act of replacing one occurrence of one redex in $X$ by its contractum. A reduction is a finite or infinite sequence of successive contractions.

The residuals of one redex when another is contracted are defined as usual (see [4, §4B] or [8, $\S \S 6$ and 9] for details). The aim of the definition is to get Property D, namely that if $\xi, \eta$ are redexes in a term $X$, then contracting $\xi$ and afterwards reducing all the residuals of $\eta$ will produce the same result as contracting $\eta$ and afterwards reducing the residuals of $\xi$; and this aim determines the definition almost uniquely.

Two redexes $\xi, \eta$ in a term $X$ will be called too close together iff one contains the other and the pair has one of the following two forms: either

$$
(\lambda x \cdot M x) N, \quad \lambda x \cdot M x
$$

or

$$
(\lambda x \cdot(\lambda y \cdot M) x), \quad(\lambda y \cdot M) x .
$$

A set of redexes in a term $X$ will be called well-separated iff no two of its 
members are too close together. Note that if $\xi$ is too close to $\eta$ and one of them is contracted, then the other has no residual; and contracting one produces the same result as contracting the other.

A set of (occurrences of) redexes which are all in the same term $X$ will be called coinitial (because when contractions are pictured as directed lines and terms as points, coinitial contractions are a bundle of lines starting at the same point). A development of a coinitial set $\alpha$ is a reduction whose contractions are residuals of the set; it is a complete development $(C D)$ of $\alpha$ iff no residuals of $\alpha$ occur in its end-term. A minimal complete development $(M C D)$ is a $C D$ whose each contraction is a minimal residual of $\alpha$.

The following notation will also be used:

$A, B, \ldots, X, Y, Z$ : Terms or occurrences of terms.

$\xi, \eta, \zeta:$ (Occurrences of) redexes.

$\rho, \sigma, \tau:$ Reductions, finite or infinite.

$\alpha, \beta$ : Coinitial sets of redexes.

$\xi / \eta$ : The set of all the residuals of $\xi$ when $\eta$ is contracted.

$\xi / \rho$ : The set of all residuals of $\xi$ in the end-term of $\rho$.

$\alpha / \rho$ : The union of all $\xi / \rho$, for all $\xi$ in $\alpha$.

$\xi / / \eta$ : Any MCD of the set $\xi / \eta$.

$\rho+\sigma: \rho$ followed by $\sigma$ (defined iff $\rho$ is finite and ends at the start of $\sigma$ ).

Finally, if $\rho, \sigma$ are two reductions, $\rho$ is weakly equivalent to $\sigma(\rho \approx \sigma)$ will mean that $\rho, \sigma$ are both finite, and $\rho$ has the same start and end as $\sigma$. Then $\rho$ is strongly equivalent to $\sigma(\rho \cong \sigma)$ will mean that $\rho$ is weakly equivalent to $\sigma$ and also for every redex $\zeta$ coinitial with $\rho$ and $\sigma$ we have

$$
\zeta / \rho=\zeta / \sigma \text {. }
$$

LEMMA 1. Every set of coinitial redexes has a complete development, in fact an $M C D$.

Proof. All terms are finite, so every coinitial set is finite; and contracting a minimal redex in a set reduces the number of residuals.

LEMMA 2. The one-step Church-Rosser property (property D mentioned above) holds, namely

$$
\xi+\eta / / \xi \simeq \xi / / \eta
$$

PRoof. Check through the cases in the definition of residuals. [4, §4B].)

LEMMA 3. For $\beta$-reduction and $\eta$-reduction (but not for $\beta \eta$-reduction) Curry's strong form of $\mathrm{D}$ holds, namely

$$
\xi+\eta / / \xi \simeq \eta+\xi / / \eta
$$

also a strong form of Curry's property $\mathrm{E}$ holds, namely 
$\left(\mathrm{E}^{+}\right) \quad$ for all coinitial sets $\alpha$, all $C D$ s of $\alpha$ are strongly equivalent.

Proof. See [4, §4]. Note that $\mathrm{D}^{+}$is a special case of $\mathrm{E}^{+}$.

LEMMA 4. For $\beta \eta$-reduction: if $\alpha$ is well-separated and $\rho$ is any finite reduction coinitial with $\alpha$, then the set $\alpha / \rho$ of residuals will be well-separated.

Proof. See [8, §9, Lemma 3].

LEMMA 5. For $\beta \eta$-reduction: property $\mathrm{E}^{+}$holds for all well-separated sets, namely all CDs of a well-separated set are strongly equivalent. As a special case, $\mathrm{D}^{+}$above holds for well-separated pairs $\xi, \eta$.

Proof. See [8, §9, Lemma 4] and following.

LEMMA 6. Any set $S$ of coinitial reductions whose lengths are all less than a number $p$ must be finite.

Proof. This lemma depends on no special properties of reductions at all, except that all terms are finite. It follows immediately from König's lemma that a finitely branching infinite tree must have at least one infinite linear branch. But the direct proof below by induction on $p$ is more finitary.

If $p=1$, then the only member of $S$ is 0 , the empty reduction.

If $p>1$, then each nonempty member $\rho$ of $S$ must have form $\rho^{\prime}+\xi$, where $\rho^{\prime}$ has less than $p-1$ contractions and $\xi$ is a single contraction. Let $S^{\prime}$ be the set of all $\rho^{\prime}$, for all nonempty $\rho$ in $S$. Then $S^{\prime}$ is finite, by the inductionhypothesis. Let $\sigma_{1}, \ldots, \sigma_{n}$ be the members of $S^{\prime}$ and let $Y_{1}, \ldots, Y_{n}$ be their end-terms. In each $Y_{i}$ there can only be a finite number $k_{i}$ of occurrences of redexes, so $S$ can only have $k_{1}+k_{2}+\cdots+k_{n}$ members.

\section{The finiteness theorem for pure $\lambda \beta \eta$-reduction.}

THEOREM 1. For $\lambda \beta \eta$-reduction, with or without type-restrictions, all developments are finite.

REMARK 1. As mentioned earlier, the $\beta$-case has already been proved by $\mathrm{D}$. E. Schroer in his thesis [12].

REMARK 2. If $X$ is any term and $\rho$ is any development of a set $\alpha$ of redexes in $X$, then $\rho$ is a development of the set $[X]$ of all the redexes in $X$. Theorem 1 will be proved by defining a number $p(X)$ by induction on $X$ and showing that all finite developments of $[X]$ have less than or equal to $p(X)$ steps (i.e. contractions). This implies that no infinite developments of $[X]$ exist, because any infinite development would have a finite initial segment with more than $p(X)$ steps.

A more direct proof, avoiding the rather messy construction of $p(X)$, can be given by showing that if $\rho$ is any infinite development of $[X]$, then some of 
the steps of $\rho$ must form an infinite development of some proper part of $X$. The theorem would then follow by induction on $X$, the basis being trivial. But picking out the proper part of $X$, and its infinite development in $\rho$, needs a weak version of the axiom of choice (as pointed out by Schroer in his thesis), or else König's lemma (compare the proof of Lemma 6 above). Using $p(X)$ avoids this 'nonfinitary' argument.

PROOF FOR $\beta$-REDUCTION. To show the main lines of the proof clearly, I shall deal first with $\beta$-reduction, and leave $\eta$-reduction till later.

For each $X$ a number $p(X)$ will be defined such that all developments of $[X]$ have $<p(X)$ steps. No attempt will be made to find the smallest possible $p(X)$; such an attempt might be interesting, but it would complicate the present account too much.

Basis. $X$ is an atom. The only development of $[X]$ is the empty reduction. Define $p(X)=0$.

Induction-case 1. $X=\lambda x \cdot X_{1}$. Then all members of $[X]$ are in $X_{1}$. Define $p(X)=p\left(X_{1}\right)$.

Induction-case 2. $X=X_{1} X_{2}$ but $X$ is not a redex. Define $p(X)=p\left(X_{1}\right)+$ $p\left(X_{2}\right)$.

Induction-case 3. $X=(\lambda x \cdot M) N$. By change of bound variables, we can assume that no variable is both free and bound in $X$ or any part of $X$. Now by the induction-hypothesis there exist numbers $p(M)$ and $p(N)$ bounding the lengths of all finite developments of $[M]$ and $[N]$ respectively. Hence, by Lemma 6, there can only be a finite number of different developments of $[M]$ and of $[N]$. Let $M_{1}, \ldots, M_{t}$ be all the terms to which $M$ reduces by (not necessarily complete) developments of $[M]$, and for each $i$ let $q_{i}$ be the number of free occurrences of $x$ in $M_{i}$. Then define

$$
\begin{aligned}
q^{\prime} & =\max \left\{1, q_{1}, \ldots, q_{t}\right\}, \\
p(X) & =\left(1+q^{\prime} \cdot p(N)\right) \cdot(1+p(M)) .
\end{aligned}
$$

We must now prove that all finite developments of $[X]$ have length less than or equal to this $p(X)$.

A development in which $X$ itself is not contracted cannot have more than $p(M)+p(N)$ steps, which is less than the $p(X)$ above.

Now let us consider a development in which $X$ is contracted. Such a development must have form

$$
\begin{aligned}
X & =(\lambda x \cdot M) N \\
& \gg\left(\lambda x \cdot M^{\prime}\right) N^{\prime} \text { by developments of }[M],[N], \\
& >\left[N^{\prime} / x\right] M^{\prime} \\
& \gg Y .
\end{aligned}
$$

If no residuals of $[M]$ are contracted in the part of (3) below $\left[N^{\prime} / x\right] M^{\prime}$, 
then that part of (3) consists of parallel nonoverlapping reductions, one in each substituted occurrence of $N^{\prime}$. Each of these reductions, when preceded by the reduction $N \gg N^{\prime}$, will form a development of $[N]$. There are $q$ of these parallel reductions, where $q$ is the number of free occurrences of $x$ in $M^{\prime}$. Hence the whole length of (3) must be less than or equal to

$$
p(M)+1+p(N) \cdot \max \{q, 1\} \text {. }
$$

Since $q \leqslant q^{\prime}$ and $1 \leqslant q^{\prime}$, this is less than or equal to (2).

Now suppose that at least one $[M]$-residual is contracted in the reduction below $\left[N^{\prime} / x\right] M^{\prime}$ in (3). In what follows, this $[M]$-contraction will be moved up the reduction, to produce a development with one less $[M]$-contraction below $\left[N^{\prime} / x\right] M^{\prime}$. Then repeating this moving-up process for the other $[M]$ contractions in the bottom part of (3) will give us a development of $[X]$ with no $[M]$-contractions below $\left[N^{\prime} / x\right] M^{\prime}$, which we have already seen to have length at most (4). The length of the original reduction will then be compared with the length of the new reduction.

If an $[M]$-contraction occurs below $\left[N^{\prime} / x\right] M^{\prime}$, the given development (3) must have the following form (see the unbroken lines in Figure 1):

$$
\begin{aligned}
X & =(\lambda x \cdot M) N \\
& \gg\left(\lambda x \cdot M^{\prime}\right) N^{\prime} \\
& >\left[N^{\prime} / x\right] M^{\prime} \text { by the residual of } X, \\
& \gg V \text { by reductions in } N^{\prime}-s, \\
& >W \text { by a residual, } \zeta \text {, of }[M], \\
& \gg Y .
\end{aligned}
$$

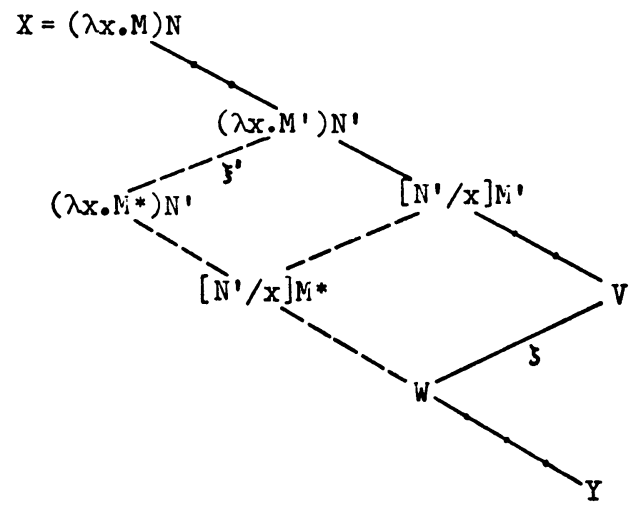

FIGURE 1

The redex $\zeta$ in $V$ is a residual of some redex $\xi$ in $M$, so $\zeta$ must be a residual of some $\xi^{\prime}$ in $M^{\prime}$ which is a residual of $\xi$ in $M$. Note that $\xi^{\prime}$ can only have one 
residual in $V$, because the reduction from $\left[N^{\prime} / x\right] M^{\prime}$ to $V$ takes place inside the substituted occurrences of $N^{\prime}$, and these $N^{\prime}$ will be either inside $\left[N^{\prime} / x\right] \xi^{\prime}$ or nonoverlapping with it.

Now replace the given reduction (5) by the following one (see the broken lines in Figure 1):

$$
\begin{aligned}
X & =(\lambda x \cdot M) N \\
& \gg\left(\lambda x \cdot M^{\prime}\right) N^{\prime} \quad \text { same as in (5), } \\
& >\left(\lambda x \cdot M^{*}\right) N^{\prime} \quad \text { by contracting } \xi^{\prime}, \\
& >\left[N^{\prime} / x\right] M^{*} \text { by the residual of } X, \\
& \gg W \text { by a reduction defined below, } \\
& \gg Y \text { same as (5). }
\end{aligned}
$$

The new reduction from $\left[N^{\prime} / x\right] M^{*}$ to $W$ is constructed as follows (essentially the same as in the proof of Curry and Feys [4, p. 113, Theorem 5], sometimes called the 'lemma of parallel moves'). In $\left[N^{\prime} / x\right] M^{\prime}$ the residuals of $[N]$ form a set $\beta$, each member lying in a substituted $N^{\prime}$; the given reduction from $\left[N^{\prime} / x\right] M^{\prime}$ to $W$ in (5) has form

$$
\left[N^{\prime} / x\right] M^{\prime}=V_{0}>V_{1}>\cdots>V_{m}=V>W,
$$

where $V_{i}>V_{i+1}$ is the contraction of a residual $\eta_{i}$ of $\beta$, and $V>W$ is the contraction of the one and only residual of $\left[N^{\prime} / x\right] \xi^{\prime}$ in $V$. Now each $V_{i}$ contains exactly one residual $\xi_{i}$ of $\left[N^{\prime} / x\right] \xi^{\prime}$; let $Z_{i}$ be the result of contracting this $\xi_{i}$ (see the unbroken lines in Figure 2). By Lemma 2 (i.e. property D) applied to the redexes $\xi_{i}$ and $\eta_{i}$ in $V_{i}$, we have

$$
Z_{i} \gg Z_{i+1}
$$

by a complete development of the residuals $\eta_{i} / \xi_{i}$ in $Z_{i}$ (see the broken lines in Figure 2). Hence we have a reduction

$$
\left[N^{\prime} / x\right] M^{*}=Z_{0} \gg Z_{1} \gg \cdots \gg Z_{m}=W,
$$

and each step of this reduction is a contraction in a substituted $N^{\prime}$ in $\left[N^{\prime} / x\right] M^{*}$. This is the reduction required for (6).

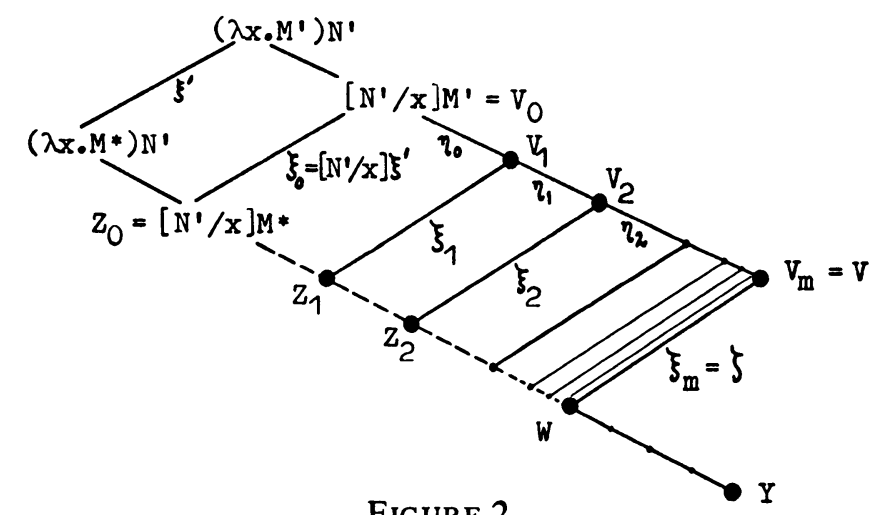

FigURE 2 
Now (8) is a development of the residuals of $[N]$ in $\left[N^{\prime} / x\right] M^{*}$. To see this, first note that by Lemma 3 (property $\mathrm{D}^{+}$) applied to the redexes $\left(\lambda x \cdot M^{\prime}\right) N^{\prime}$ and $\xi^{\prime}$ (see Figure 1), the residuals of $[M]$ and $[N]$ in $\left[N^{\prime} / x\right] M^{*}$ are the same with respect to both the reductions

$$
\begin{aligned}
& X \gg\left(\lambda x \cdot M^{\prime}\right) N^{\prime}>\left[N^{\prime} / x\right] M^{\prime}>\left[N^{\prime} / x\right] M^{*}, \\
& X \gg\left(\lambda x \cdot M^{\prime}\right) N^{\prime}>\left(\lambda x \cdot M^{*}\right) N^{\prime}>\left[N^{\prime} / x\right] M^{*} .
\end{aligned}
$$

Hence in $\left[N^{\prime} / x\right] M^{*}$ (see Figure 2), the residuals $\eta_{0} / \xi_{0}$, which are residuals of $[N]$, are also residuals of $[N]$ with respect to the new reduction (10); so the new reduction from $X$ to $Z_{1}$ is a development of $[X]$.

Next, applying Lemma 3 (property $\mathrm{D}^{+}$) to $\xi_{0}$ and $\eta_{0}$ in $V_{0}$, then to $\xi_{1}$ and $\eta_{1}$ in $V_{1}$, and so on, shows us that for each $i$ the residuals of $\eta_{i}$ in $Z_{i}$ are residuals of $[N]$ also with respect to the new reduction to $Z_{i}$. Hence the whole new reduction (8) is a development of the residuals of $[N]$.

Furthermore, by the same argument, the residuals of $[X]$ in $W$ at the end of (8) are the same as the [X]-residuals in $W$ after the original reduction in (5). Hence the new reduction (6) is a development of $[X]$.

Thus we have replaced (5) by a new development (6) with one less $[M]$-contraction below $\left[N^{\prime} / x\right] M^{\prime}$. Repeating the above replacement-process again and again will eventually give us a development with no $[M]$-steps below $\left[N^{\prime} / x\right] M^{\prime}$. In fact, we must reach such a reduction after at most $p(M)$ applications of the replacement-process, because each time the process is used, it adds one extra step to the given development $M \rightarrow M^{\prime}$ at the start of (5).

Now, how do the lengths of (5) and (6) compare? Both reductions have the same number of $[M]$-steps. But for $[N]$-steps, $(5)$ has

$$
\left[N^{\prime} / x\right] M^{*}=V_{0}>V_{1}>\cdots>V_{m}=V,
$$

whereas (6) has (see Figure 2)

$$
\left[N^{\prime} / x\right] M^{*}=Z_{0} \gg Z_{1} \gg \cdots \gg Z_{m}=W .
$$

Each step in (11) is in a substituted occurrence of $N^{\prime}$, and is part of a development of $[N]$. The number of substituted $N^{\prime \prime}$ s is the number, $q$, of free $x$ 's in $M^{\prime}$. Hence we have

$$
\text { length of }(11)<q \cdot p(N)<q \cdot p(N)+\text { length of }(12),
$$

independently of the length of (12). Therefore

$$
\text { length of (5) }<q \cdot p(N)+\text { length of (6) } \leqslant q^{\prime} \cdot p(N)+\text { length of (6), }
$$

where $q^{\prime}$ was defined by (1).

Since the replacement of (5) by (6) can be repeated at most $p(M)$ times, we get

$$
\text { length of }(5) \leqslant p(M) \cdot q^{\prime} \cdot p(N)+h \text {, }
$$


where $h$ is the maximum possible length of a development with no [M]contractions in it below $\left[N^{\prime} / x\right] M^{\prime}$. By the line above (4),

$$
h \leqslant p(M)+1+p(N) \cdot q^{\prime} \text {. }
$$

Hence the length of (5) must be less than or equal to

$$
\left(1+q^{\prime} \cdot p(N)\right) \cdot(p(M)+1),
$$

which is the $p(X)$ defined in (2). This proves the theorem in the $\lambda \beta$-case.

ProOF FOR $\beta \eta$-REDUCTION. To prove Theorem 1 for $\beta \eta$-reduction we modify the $\beta$-proof as follows.

Induction-case 1. $X=\Lambda x \cdot X_{1}$. If all the members of [X] are in $X_{1}$, define $p(X)=p\left(X_{1}\right)$ just as in the $\beta$-proof. But now there is the possibility that $X$ is an $\eta$-redex,

$$
X=\lambda x \cdot M x
$$

In this case define $p(X)=1+p(M x)$.

Any development of $[X]$ which involves the residual of $X$ must have form

$$
\begin{aligned}
X & =\lambda x \cdot M x \\
& \gg \lambda x \cdot M^{\prime} x \text { by a reduction in } M \\
& >M^{\prime} \text { by } \eta \text {-contraction } \\
& \gg Y .
\end{aligned}
$$

Now it might be that $M x$ is a redex; but if it is, it must be a $\beta$-redex, and any contraction of $M x$ or its residual would leave $\lambda x \cdot M x$ without any residual. So in the above reduction, since $\lambda x \cdot M x$ is contracted, $M x$ cannot be contracted before the stage $\lambda x \cdot M^{\prime} x$. And it cannot be contracted after that stage either, because in $M^{\prime}$ it has no residual.

In the above reduction the steps $M \gg M^{\prime} \gg Y$ form a development of $M$, so the total length is less than or equal to $1+p(M)$. Now by the various cases of the definition of $p$, we have $p(M) \leqslant p(M x)$, so $1+p(M) \leqslant p(X)$, as required. This completes Induction-case 1 .

Induction-case 2. $X=(\lambda x \cdot M) N$. Define $p(X)$ by (2), just as in the $\beta$-proof.

Any development in which $X$ itself is not contracted must have length at most $p(\lambda x \cdot M)+p(N)$, which is less than or equal to (2) because $p(\lambda x \cdot M)$ $<1+p(M)$.

Now consider a development in which $X$ is contracted. If $\lambda x \cdot M$ is an $\eta$-redex, its residual cannot be contracted in this development, because contracting either of $(\lambda x \cdot M) N, \lambda x \cdot M$, destroys the residual of the other. Hence the development has the form (3), just as before, and can be replaced by (6) just as before.

The proof that (6) is a development of $[X]$ is just like the $\beta$-proof, except 
that where that proof used Lemma 3 (i.e. property $\mathrm{D}^{+}$), the present proof must use Lemma 5 instead.

Lemma 5 requires that its $\xi$ and $\eta$ be well-separated. In the first place where Lemma 5 is needed, namely in Figure 2 for the redexes

$$
\left(\lambda x \cdot M^{\prime}\right) N^{\prime}, \quad \xi^{\prime},
$$

this condition is satisfied because $\xi^{\prime}$ is part of $M^{\prime}$.

The separation condition is also satisfied in the later applications of the lemma, namely to the pairs $\xi_{i}, \eta_{i}$ in $V_{i}(i=0, \ldots, m-1)$. To see this, note that $\xi_{i}$ is a residual of $\left[N^{\prime} / x\right] \xi^{\prime}$ in $\left[N^{\prime} / x\right] M^{\prime}$, and $\eta_{i}$ is a residual of a redex in a substituted $N^{\prime}$. The redex $\xi^{\prime}$ must have one of the forms

$$
\lambda z \cdot U_{1} z, \quad\left(\lambda z \cdot U_{1}\right) U_{2} .
$$

Substituting $N^{\prime}$ into $\xi^{\prime}$ will put occurrences of $N^{\prime}$ into $U_{1}$ and $U_{2}$, and hence $\eta_{i}$ will be in some term $U_{j}^{\prime}$ to which $\left[N^{\prime} / x\right] U_{j}$ reduces, for $j=1$ or 2 . And $\xi_{i}$ will have one of the forms

$$
\lambda z \cdot U_{1}^{\prime} z, \quad\left(\lambda z \cdot U_{1}^{\prime}\right) U_{2}^{\prime} .
$$

So $\eta_{i}$ cannot be too close to $\xi_{i}$.

This completes the proof of Theorem 1.

4. Combinatory weak reductions. The basic notation for combinatory terms may be found in [4, Chapter 5] or [10, Chapter 2]. Combinatory weak reduction, using the atomic combinators $\mathbf{S}$ and $\mathbf{K}$, is defined by the axioms

$$
\mathrm{S} X Y Z>X Z(Y Z), \quad \mathrm{K} X Y>X \text {. }
$$

These will be regarded here as an infinity of axioms, generated from two axiom-schemes

$$
\mathbf{S} v_{1} v_{2} v_{3}>v_{1} v_{3}\left(v_{2} v_{3}\right), \quad \mathbf{K} v_{1} v_{2}>v_{1},
$$

by substituting particular terms for the variables $v_{1}, v_{2}, v_{3}$. These variables will be called meta-variables to emphasize their special purpose.

The definitions of 'redex', 'residual', etc. for combinatory weak reduction are similar to those for $\lambda$-reduction. And Lemmas 1-3 and 6 can easily be proved for combinators. (See [8, $\$ \$ 6$ and 9].)

THEOREM 2. For combinatory weak reduction, with or without typerestrictions, all developments are finite.

Proof. One way is to follow the $\lambda$-proof. Another way is to deduce Theorem 2 as a special case of Theorem 3(i) in the next section. (See the proof of Theorem 3(ii).)

REMARK. Both the above proofs would still be valid for any other choice of the basic combinators; in fact, for any other set of operators satisfying (D1)-(D7) below. 
5. Reductions with extra operators. Often when a $\lambda$ - or combinatory system is used in the literature, extra operators are included in the definition of reduction. For example, when representing the primitive recursive functions of finite type in a typed $\lambda$-system with zero and successor adjoined as atoms (cf. [10, Chapter 11]), one needs to adjoin recursion operators $\mathbf{R}$ as new atoms, with axioms of the form

$$
\begin{aligned}
\mathbf{R} U_{1} U_{2} \mathbf{0} & >U_{1}, \\
\mathbf{R} U_{1} U_{2}(\boldsymbol{\sigma} \mathbf{n}) & >U_{2} \mathbf{n}\left(\mathbf{R} U_{1} U_{2} \mathbf{n}\right) .
\end{aligned}
$$

(Here $\mathbf{0}$ and $\boldsymbol{\sigma}$ are the atomic constants for zero and successor, and $\mathbf{n}$ is $\boldsymbol{n} \boldsymbol{\sigma}$ 's applied to $\mathbf{0}$ in the usual way.)

It is natural to ask whether the finiteness theorem and other basic reduction-theorems hold for this modified reduction.

For typed terms and the recursion-operators the answer is very strong; all reductions, not only developments, are finite. But to prove this needs transfinite induction, and it is still interesting to ask for a proof for developments which only need $\omega$-induction.

For terms not restricted by types, one can still adjoin a recursion operator or other similar operators and ask whether the finiteness theorem remains true. In fact it does, as we shall see below. And the class of operators which may be added is rather wide.

In what follows I shall assume that a finite or infinite sequence $a_{1}, a_{2}, \ldots$ of new atomic constants (not necessarily all distinct) has been added to the definition of ' $\lambda$-term' or 'combinatory term'. Each $a_{i}$ is assumed to have an associated axiom-scheme of the form

$$
a_{i} M_{i 1} \cdots M_{i n_{i}}>N_{i}
$$

where the $M$ 's and $N$ are terms which may contain, besides variables and $a$ 's and other constants, extra atoms $v_{1}, v_{2}, \ldots, v_{k_{1}}$ called meta-variables. The result of substituting for these meta-variables terms $U_{1}, \ldots, U_{k_{1}}$ not containing meta-variables, will be called an axiom. Hence each axiom has form

$$
a_{i} M_{i 1}^{\prime} \cdots M_{i n_{1}}^{\prime}>N_{i}^{\prime},
$$

where $X^{\prime}=\left[U_{1} / v_{1}\right] \ldots\left[U_{k_{1}} / v_{k_{i}}\right] X$ for all $X$.

In a type-restricted system, (14) is only called an axiom when the $U$ 's are such that the left-hand side and the right-hand side of (14) are well-typed.

EXAMPLE 1. The recursion operator, $\mathbf{R}$, when adjoined to untyped terms, has the axiom-schemes

$$
\begin{aligned}
\mathbf{R} v_{1} v_{2} \mathbf{0} & >v_{1}, \\
\mathbf{R} v_{1} v_{2}(\boldsymbol{\sigma n}) & >v_{2} \mathbf{n}\left(\mathbf{R} v_{1} v_{2} \mathbf{n}\right),
\end{aligned}
$$


where $\mathbf{0}, \boldsymbol{\sigma}$ and $\mathbf{n}$ are as in (13). To make these schemes fit the form (a), we must consider them as an infinite sequence of axiom-schemes, one for each $n>0$, and take

$$
a_{1}=a_{2}=\cdots=\mathbf{R} \text {. }
$$

In a type-restricted system one would not adjoin one $\mathbf{R}$ but an infinity of $\mathbf{R}$ 's, each with a different type. All these R's can still be regarded as a sequence $a_{1}, a_{2}, \ldots$ with an infinity of repetitions.

EXAMPLE 2. The iterator, $\mathbf{Z}$, when adjoined to untyped terms, has an infinity of axioms, one for each $n \geqslant 0$, thus:

$$
\mathbf{Z n}>\lambda x y \cdot x(x(\cdots(x y) \cdots)),
$$

where there are $n x$ 's applied to the $y$ on the right (For combinatory terms ' $\lambda x y$ ' means ' $[x, y]$ ' as defined by any of the usual algorithms, for example [10, p. 18, Definition 2.10].) These Z-axioms can be viewed as special cases of (a) in which no meta-variables occur, the axioms being the same as the axiom-schemes. To do this, we would take

$$
a_{1}=a_{2}=\cdots=\mathbf{Z} \text {. }
$$

In a type-restricted system one would adjoin not one $\mathbf{Z}$ but an infinity of $\mathbf{Z}$ 's, each with a different type and its own infinite sequence of axioms, which can still be regarded as special cases of $(a)$.

EXAMPLE 3. Curry's generalized $\delta$-conversions, of [4, p. 93], are axioms with the form

$$
a M_{1} \cdots M_{n}>N
$$

such that

(i) the $M$ 's contain no free variables and no redexes of any kind;

(ii) no term can be the left-hand side of two axioms (17);

(iii) for no $k<n$ is $a M_{1} \ldots M_{k}$ a redex of any kind;

(iv) $N$ contains no free variables.

Curry's conditions say in effect that no $\delta$-redex contains another redex as a proper part. (Here redex means the left-hand term of an axiom.) The conditions are satisfied by Church's $\delta$-conversion [4, p. 94], and by the $\mathrm{Z}$-axioms above. But they are not satisfied by the R-axioms, because in (13) the $U$ 's could contain other redexes. Any axioms (17) can be regarded as a special kind of axiom-schemes $(a)$ in which no meta-variables occur, just as with $\mathbf{Z}$ above.

EXAMPLE 4. The fixed-point operator, Y. When terms are not restricted by types, $\mathbf{Y}$ is definable. But in a typed system, if we want $\mathbf{Y}$ we must adjoin it as a new atom, with its own axiom-scheme. Or, more precisely, we must adjoin a new atom $Y_{\tau}$ for each type $\tau$ (and give it the type $((\tau \rightarrow \tau) \rightarrow \tau)$, in an obvious notation), and postulate 


$$
\mathbf{Y}_{\tau} v_{1}>v_{1}\left(\mathbf{Y}_{\tau} v_{1}\right) \text {. }
$$

These axiom-schemes can be regarded as special cases of $(a)$. (Note incidentally that reduction with (18) does not have the property that all reductions are finite, in contrast to the typed versions of $\mathbf{R}$ and $\mathbf{Z}$.)

Instead of $\mathbf{Y}_{\tau}$, we could adjoin for each constant term $X$ a separate atom $\mathbf{Y}_{X}$, a fixed point of $X$, with the axiom

$$
\mathbf{Y}_{\boldsymbol{X}}>\boldsymbol{X} \mathbf{Y}_{\boldsymbol{X}} .
$$

These axioms also can be regarded as special cases of $(a)$.

General notation. The left-hand side of an (a)-atom (14) will be called an $(a)$-redex or $a_{i}$-redex, and the corresponding right-hand side its contractum. Other notation will be the same as in $\$ 2$, but referring now to reduction defined by adding (a)-schemes to the $\lambda$ - or combinatory schemes. But residuals cannot be defined without the assumptions below.

Assumptions. All axiom-schemes (a) to be discussed in future will be assumed to satisfy conditions (D1)-(D7) below. These conditions have been motivated and discussed in [7, §8] and $[8, \S 6]$; their main purpose is to ensure that

(i) contracting an (a)-redex does not violate any type-restrictions which may be in the system,

(ii) each redex has a unique contractum,

(iii) contracting a small redex inside a larger one leaves the larger one still a redex,

(iv) residuals are definable in such a way that Property $D$ (Lemma 2) holds. Conditions (D1)-(DT) will easily be seen to be satisfied by $\mathbf{R}, \mathbf{Z}, \mathbf{Y}_{\tau}, \mathbf{Y}_{X}$, and any generalized $\delta$-conversions satisfying the conditions of Example 3.

(D1) If type-restrictions are present, contracting an (a)-redex in a term does not change the term's type.

(D2) A redex cannot be an instance of the left side of two (a)-schemes.

(D3) Each meta-variable in $N_{i}$ on the right of (a) also occurs on the left of (a).

(D4) No meta-variable occurs twice on the left side of (a).

(D) If an $a_{k}$-redex $P$ is a proper part of an $a_{i}$-redex $Q$, then $P$ is in one of the U's that were substituted for the meta-variables.

(D6) No meta-variable occurs in function-position in the left side of (a), and (in $\lambda$-systems): no $\beta$-redex occurs in the left side of (a); (in combinatory systems): no combinatory redex occurs in the left side of $(a)$ and no $a_{i}$ is an atomic combinator.

(D7) $(\lambda$-systems only): No scheme (a) contains free variables (other than meta-variables), and if (a) contains a part $\lambda x \cdot Y$, then $Y$ contains no metavariables and (a) stays valid after changes of bound variables. 
In (D6), 'function position' means the position of the $v$ in a component with form $(v X)$. In (D7), 'stays valid after changes of bound variables' means that if $P^{\prime}>Q^{\prime}$ results from changing bound variables ( $\alpha$-conversion) in an (a)-axiom-scheme $P>Q$, then $P^{\prime}>Q^{\prime}$ has also been included in the list of (a)-axiom-schemes. Any set of schemes satisfying all the conditions except this one clause can obviously be made to satisfy this too by adding an infinite number of 'copies' of the schemes already in it.

If $\eta$-contractions are not involved, (D1)-(D7) are enough to ensure that redexes do not 'interfere' with each other ((iii) above), without any more assumptions being needed. (See $[7, \S 8]$, and $[8, \S 6]$.) But when $\eta$-contractions are present, the following two extra conditions will be assumed (see the start of $[8, \S 9]$, or $[7, \S 9$, p. 15$]$, for details):

(D8) In the left side of (a), the last argument $M_{i n_{i}}$ is not a single metavariable.

(D9) No $M_{i j}$ in (a) contains an $\eta$-redex.

These two conditions are obviously satisfied by $\mathbf{R}$ and $\mathbf{Z}$ and the fixedpoints $\mathbf{Y}_{\boldsymbol{X}}$. But they are not satisfied by the other operators $\mathbf{Y}_{\boldsymbol{\tau}}$. I do not know if this failure of (D8)-(D9) leads to a failure of the finiteness theorem for $\beta-\eta-Y_{\tau}$-reduction, but it does make other desirable theorems fail. For instance, a counterexample to the Church-Rosser theorem is

$$
\mathbf{Y}_{\tau}<_{\eta} \lambda x \cdot \mathbf{Y}_{\tau} x>\lambda x \cdot x\left(\mathbf{Y}_{\tau} x\right) .
$$

(In contrast, this theorem does not fail for the fixed-points $\mathbf{Y}_{X}$, since it is a consequence of (D1) - (D9).) It is also interesting that if the $\mathbf{R}$-schemes were only slightly modified from (15), then (D8)-(D9) and the Church-Rosser theorem would fail. (See [7, §9, p. 15].)

LEMMAS 1 To 6 EXTENDED. Assuming (D1)-(D7), and also (D8)-(D9) when $\eta$-redexes are present, one can define residuals in a fairly obvious way. The details are in [8, $\$ \S 6$ and 9]. Well-separatedness is defined exactly as before; in particular, $(a)$-redexes are always well-separated from all others. Then Lemmas 1 to 6 all hold. In fact, Lemma 1 is obvious, Lemma 2 is proved by checking the definition of residuals in [8, $\$ \$ 6$ and 9], Lemma 3 is the main result of [8, §6], Lemma 4 is [8, $\$$, Lemma 3], Lemma 5 is proved in $[8, \S 9$, Lemma 4] and following; Lemma 6 is obvious.

THEOREM 3. All developments are finite, in the following systems, with or without type-restrictions:

(i) $\lambda \beta$-reduction modified by (a)-schemes satisfying (D1)-(D7);

(ii) combinatory weak reduction modified by (a)-schemes satisfying (D1)-(D7);

(iii) $\lambda \beta \eta$-reduction modified by (a)-schemes satisfying (D1)-(D9).

Proof OF PART (i). The proof is the same as for pure $\beta$-reduction, modified 
as follows. Recall that the aim of the proof is to define, by induction on an arbitrary term $X$, a number $p(X)$ such that all finite developments of the redexes in $X$ have $<p(X)$ contractions.

Basis. $X$ is an atom. If $X$ is not a redex, define $p(X)=0$, as before. If $X$ is an $a_{i}$ which is itself a redex, define $p(X)=1$.

New induction-case 4. $X$ is an (a)-redex. Suppose that $a M_{1} \cdots M_{n}>N$ is an axiom-scheme, and

$$
X=\left[U_{1} / v_{1}\right] \cdots\left[U_{k} / v_{k}\right] M .
$$

Then the redexes in $X$ are $X$ itself together with redexes in the substituted occurrences of $U_{1}, \ldots, U_{k}$. By (D4), there is only one such occurrence of each $U_{j}$ in $X$. But there may be more in the contractum, depending on how many times each $v_{j}$ occurs in $N$. Let $m_{j}$ be the number of occurrences of $v_{j}$ in $N$, and define

$$
\begin{gathered}
m_{j}^{\prime}=\max \left\{m_{j}, 1\right\}, \\
p(X)=1+m_{1}^{\prime} \cdot p\left(U_{1}\right)+m_{2}^{\prime} \cdot p\left(U_{2}\right)+\cdots+m_{k}^{\prime} \cdot p\left(U_{k}\right) .
\end{gathered}
$$

Now consider any finite development of the redexes in $X$. If $X$ itself is not contracted, the development will take place entirely inside the substituted $U_{1}, \ldots, U_{k}$ in $X$, and so its length will be less than or equal to

$$
p\left(U_{1}\right)+\cdots+p\left(U_{k}\right)
$$

which is less than or equal to $p(X)$.

On the other hand, if $X$ is contracted then the development must have form

$$
\begin{aligned}
X & =\left[U_{1} / v_{1}\right] \cdots\left[U_{k} / v_{k}\right]\left(a M_{1} \cdots M_{n}\right) \\
& \gg\left[U_{1}^{*} / v_{1}\right] \cdots\left[U_{k}^{*} / v_{k}\right]\left(a M_{1} \cdots M_{n}\right) \\
& >\left[U_{1}^{*} / v_{1}\right] \cdots\left[U_{k}^{*} / v_{k}\right] N \\
& \gg \cdots
\end{aligned}
$$

The last part of the development will consist of reductions inside substituted occurrences of $U_{i}^{*}, \ldots, U_{k}^{*}$. Each $U_{j}^{*}$ will have $m_{j}$ such occurrences, and each occurrence may be reduced. Consider any one of these reductions: together with the reduction from $U_{j}$ to $U_{j}^{*}$, it will form a development of $\left[U_{j}\right]$ :

$$
U_{j} \gg U_{j}^{*} \gg \cdots \text {. }
$$

By the induction-hypothesis the length of this development cannot be more than $p\left(U_{j}\right)$. Hence the total number of steps in the given development of $[X]$ cannot be more than

$$
1+m_{1}^{\prime} \cdot p\left(U_{1}\right)+\cdots+m_{k}^{\prime} \cdot p\left(U_{k}\right)
$$

which is $p(X)$.

Proof of PART (ii). The combinatory terms can be regarded as $\lambda$-terms containing no $\lambda$ 's, and $S$ and $K$ and $a_{1}, a_{2}, \ldots$ can be considered as new 
atoms added to the $\lambda \beta$-calculus. If $a_{1}, a_{2}, \ldots$ satisfy (D1)-(D7) with respect to $S$ and $K$, then $S, K, a_{1}, a_{2}, \ldots$ can easily be seen to satisfy (D1)-(D7) with respect to $\lambda \beta$-reduction. Hence part (ii) is a special case of (i).

Proof OF PART (iii). The proof is the same as for pure $\beta \eta$-reduction, but with the extra basis and induction cases given above for (i).

\section{REFERENCES}

1. H. P. Barendregt, J. Bergstra, J. W. Klop and H. Volken, Degrees, reductions and representability in the lambda calculus, Math. Instituut, Budapestlaan 6, Utrecht, preprint 22, 1976.

2. A. Church and J. B. Rosser, Some properties of conversion, Trans. Amer. Math. Soc. 39 (1936), 472-482.

3. H. B. Curry, A study of generalized standardization in combinatory logic, Lecture Notes in Math., vol. 500, Springer-Verlag, Berlin and New York, 1974, pp. 44-55.

4. H. B. Curry and R. Feys, Combinatory logic. I, North-Holland, Amsterdam, 1958. MR 20 \#817.

5. H. B. Curry, R. Hindley and J. P. Seldin, Combinatory logic. II, North-Holland, Amsterdam, 1972.

6. R. Hindley, An abstract form of the Church-Rosser theorem. I, J. Symbolic Logic 34 (1969), 545-560. MR 46 \# 1578.

7. 1-21. MR 50 \#61.

8. 227-248.

9. $\_$Standard and normal reductions, Trans. Amer. Math. Soc. (to appear).

10. R. Hindley, B. Lercher and J. P. Seldin, Introduction to combinatory logic, Cambridge Univ. Press, London and New York, 1972. MR 49 \#25.

11. J. M. E. Hyland, A simple proof of the Church-Rosser theorem, unpublished manuscript available from J. Hyland, Math. Inst., 24-29 St. Giles, Oxford, England.

12. D. E. Schroer, The Church-Rosser theorem, Doctoral thesis, Cornell University, Ithaca, N. Y., 1965.

Department of Pure Mathematics, University College, Swansea, Wales, Unted KINGDOM SA2 8PP 